\title{
Genetic analysis of families of patients with Behçet's syndrome: data incompatible with autosomal recessive inheritance
}

\author{
J A BIRD STEWART
}

From the Department of Genetics, University of Leeds

SUMmARY Data on families with Behçet's syndrome were analysed to test a hypothesis of autosomal recessive inheritance. Fifteen families from the UK and nine from Turkey were included. There were 27 individuals with Behçet's syndrome according to the Japanese criteria ${ }^{10}$ and 119 unaffected individuals. There were no affected parents in the families. The 'goodness of fit' test of Elandt-Johnson ${ }^{11}$ was applied to the distribution of affected individuals in these families, and the data were found to be incompatible with autosomal recessive inheritance. HLA associations were also examined in patients and relatives, and this led to re-examination of previously published data on HLA associations with Behçet's syndrome.

Key words: genes-recessive, statistics, pedigree, HLA antigens.

Familial aggregation of Behçet's syndrome has been reported in the literature. In one Japanese study ${ }^{1}$ eight of 223 patients had affected first degree relatives, and two had affected second degree relatives. These 10 patients were clustered in five families. The other 213 patients appeared as sporadic cases with unaffected parents. All other reports of familial Behçet's syndrome are accounts of isolated kindred ${ }^{2-7}$ and show no consistent inheritance pattern. A similar survey by Chamberlain $^{8}$ of 32 patients in the UK showed an increase in symptoms associated with Behçet's syndrome in first degree relatives, but none of these relatives had Behçet's syndrome according to the criteria of Mason and Barnes. ${ }^{2}$

The existence of a possible familial component has been thought to be important for the aetiology of this disease, e.g., a family history of Behçet's syndrome is one of the minor criteria of Mason and Barnes diagnostic classification; ${ }^{2}$ Aoki suggests that the finding of familial aggregations militates against an infectious aetiology for Behçet's syndrome because the onset in relatives is widely separated in time and space. ${ }^{1}$ The reports of HLA associations, which are now well documented, are often taken to support a genetic aetiology. ${ }^{9}$

Accepted for publication 11 September 1985.

Correspondence to Dr J A Bird Stewart, Department of Genetics, University of Leeds. Leeds LS2 9JT.
However, Behçet's syndrome does not show a consistent inheritance pattern of any classic Mendelian type. The majority of cases appear to be sporadic with unaffected parents. It could be suggested that this might be consistent with an autosomal recessive pattern of inheritance. Such a mode of inheritance would lead to a definite distribution of numbers of affected and unaffected siblings in families of different sizes. This paper presents an appropriate statistical test of the expectations for autosomal recessive inheritance in a group of families from the UK and a group from Turkey.

\section{Materials and methods}

The groups of patients were subjected to a family survey of the type described by Chamberlain ${ }^{8}$ except that no examination was undertaken for the presence of sacroiliitis. Patients and relatives were interviewed and examined, and the results recorded on a standard pro forma. Histocompatibility antigen data were available for all patients and relatives. Fifteen families were from the UK and nine families were from Turkey. Two additional Turkish families were excluded because there was no information on parents. Diagnosis of affected individuals in the families, made on the basis of the Japanese criteria, ${ }^{10}$ is given in Table 1 . A summary of the clinical features in all patients is given in Table 3. 
Because families are ascertained through an affected proband, any test for a genetic aetiology based on expected proportions of affected children must contain a correction for the unascertained families where all children are unaffected. There would otherwise be an overestimate of the proportion of affected individuals. A suitable method is given by Elandt-Johnson. ${ }^{11}$ The necessary computations are shown in Table 2. This 'goodness of fit' test has been used to demonstrate heterogeneity in data on the occurrence of microcephaly, caused by the inclusion of cases due to infection and cases due to recessive inheritance in the same sample. The use of this approach is, therefore, doubly valuable because it not only helps to decide if the data fit a particular model but also indicates heterogeneity due to mixed aetiologies. Two special statistics are computed, $Z^{2}$ combined and $Z^{2}$ difference. They are distributed as $\chi^{2}$, and 'goodness of fit' to the model is shown when $Z^{2}$ comb is non-significant. That is to say, the distribution of affected children in the families is compatible with the genetic hypothesis under test, and there is, therefore, no significant difference between the observed and expected figures. However, if $\mathrm{Z}^{2}$ diff is significant heterogeneity in the data is indicated, so that a fit to the mode of inheritance under consideration can only be accepted if both of these quantities are nonsignificant.

Frequencies of HLA antigens in patients, controls, and relatives were also examined. Because of the relatively small numbers a Fisher's exact probability was calculated instead of the usual $\chi^{2}$ or variant of it.

\section{Results}

The distribution of clinical features of patients in this study was substantially similar to those of previous findings. Mouth ulcers (MU) were found in all 27 patients, genital ulcers (GU) in $22(81 \%)$, eye inflammation in $14(52 \%)$, and arthritis/arthralgia in $18(67 \%)$, while the gastrointestinal tract (GI) and central nervous system (CNS) were more rarely affected (Table 3). The majority of pedigrees contained one affected individual only ( 21 out of 24 families), but three families had two affected members. None had more. There was a total of 119

Table 1 Diagnosis of individuals with Behçet's syndrome, based on the Japanese criteria ${ }^{10}$

\begin{tabular}{lllll}
\hline & Complete & Incomplete & Suspect & Total \\
\hline UK & 3 & 12 & 2 & 17 \\
Turkey & 3 & 7 & 0 & 10 \\
\hline
\end{tabular}

Table 2 Computations for Elandt-Johnson's test for 'goodness of fit' of family data to an autosomal recessive hypothesis $^{\text {II }}$

Observed values

For each family,

size, $s$

Number of families

Number of of that size, $n_{\mathrm{s}}$

affected children $r_{s} \frac{\mathscr{C}}{\vec{D}}$

\section{Calculated values}

To fit autosomal recessive model, the probability of any child being affected, $\theta=0 \cdot 25$.

For any family size, the expected number of affected children $=\mathrm{n}_{\mathrm{s}} \mathbf{A}_{\mathrm{s}}$, where

$$
A_{s}=\frac{s(\theta)}{1-(1-\theta)^{s}}
$$

The variance of affected number is $n_{s} B_{s}$, where

$$
B_{s}=\frac{s \theta(1-\theta)}{1-(1-\theta)^{s}}-\frac{s^{2} \theta^{2}(1-\theta)^{s}}{\left[1-(1-\theta)^{s}\right]^{2}}
$$

Then,

$$
\begin{aligned}
& z^{2}=\frac{\left(r_{s}-n_{s} A_{s}\right)^{2}}{n_{s} B_{s}} \text {, with } 1 \text { df. } \\
& Z_{\text {total }}^{2}=\underset{s=2}{s} z^{2} \text {, with } 1 \mathrm{df} \text { for each } z \text { value, total } x \mathrm{df} \text {. }
\end{aligned}
$$

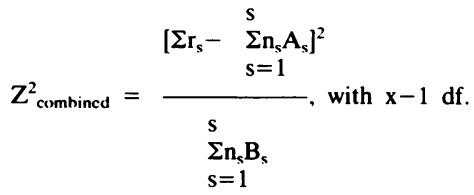

$$
\begin{aligned}
& \mathrm{Z}_{\text {diff }}^{2}=\mathrm{Z}_{\text {total }}^{2}-\mathrm{Z}_{\text {combincd, }}^{2} \text { with } 1 \mathrm{df} \text {. }
\end{aligned}
$$

unaffected relatives, and siblings ranged in number from one to eight.

Table 4 shows the results of fitting the distribution of affected individuals in families of different sizes to the expectation of an autosomal recessive pattern of inheritance. The significant value of $\mathrm{Z}_{\text {comb }}^{2}$ showed that the data did not fit an autosomal mode of inheritance. The proportion of affected children in families of different sizes was not distributed as would be expected on this hypothesis. However, the value for $\mathrm{Z}^{2}$ diff was low, suggesting an absence of heterogeneity. There is some consistent pattern in all the different family sizes. This finding prompted a question about the occurrence of minor symptoms in first degree relatives of patients with Behçet's syndrome. Features such as mouth ulcers, genital ulcers, sacroiliitis, and psoriasis have been reported in first degree relatives. ${ }^{8}$ Since data on such symptoms were available on the families reported here it seemed interesting to try a 'goodness of fit' including individuals with symptoms as 'affected'. Mouth and genital ulcers were chosen as being the 
Genetic analysis of families of patients with Behçet's syndrome

Table 3 Clinical characteristics of all 27 individuals with Behçet's syndrome in the families used in this study

\begin{tabular}{|c|c|c|c|c|c|c|c|c|c|}
\hline $\begin{array}{l}\text { Family } \\
\text { No. }\end{array}$ & $M / F$ & $M U$ & $G U$ & Iritis & $\begin{array}{l}\text { Skin } \\
\text { lesions }\end{array}$ & $G I$ & Vascular & Arthritis & CNS \\
\hline 9 & $\mathbf{M}$ & + & + & - & + & - & + & - & - \\
\hline 10 & $\mathbf{M}$ & + & + & - & + & - & + & + & - \\
\hline 11 & $\mathrm{~F}$ & + & + & - & - & - & - & + & - \\
\hline 23 & $\mathbf{F}$ & + & + & - & + & - & - & - & - \\
\hline 31 & $\mathrm{M}$ & + & + & + & + & - & - & + & + \\
\hline 42 & $\mathrm{M}$ & + & + & - & + & - & - & + & - \\
\hline 43 & M & + & + & + & - & - & - & + & - \\
\hline 50 & $\mathbf{M}$ & + & + & - & - & - & - & - & - \\
\hline 51 & $F$ & + & + & + & + & - & - & + & - \\
\hline 52 & $\mathbf{F}$ & + & + & + & + & - & + & + & - \\
\hline 53 & $\mathrm{~F}$ & + & + & + & + & - & - & + & - \\
\hline 54 & $\mathbf{F}$ & + & + & + & + & - & - & + & + \\
\hline $55 a$ & $\mathbf{F}$ & + & + & - & - & - & - & + & - \\
\hline $55 b$ & $\mathrm{~F}$ & + & + & - & + & - & - & + & - \\
\hline $56 a$ & $\mathrm{~F}$ & + & + & - & - & - & - & + & - \\
\hline $56 b$ & $F$ & + & + & + & + & - & - & + & - \\
\hline 80 & $\mathbf{M}$ & + & - & - & + & - & - & + & - \\
\hline 200 & $F$ & + & + & - & + & - & - & - & - \\
\hline 201 & $\mathbf{M}$ & + & + & - & + & - & - & - & - \\
\hline 202 & $\mathbf{M}$ & + & + & - & + & - & - & + & - \\
\hline 203 & $\mathrm{~F}$ & + & - & + & + & - & - & - & - \\
\hline $204 a$ & M & + & - & + & + & - & - & - & - \\
\hline $204 b$ & $\mathbf{M}$ & + & - & + & + & - & - & - & - \\
\hline 206 & $\mathbf{M}$ & + & + & + & - & - & - & + & - \\
\hline 207 & $\mathbf{F}$ & + & + & + & + & - & - & - & - \\
\hline 208 & $\mathbf{F}$ & + & - & + & + & - & - & + & - \\
\hline 210 & $\mathbf{M}$ & + & + & + & + & - & - & + & - \\
\hline
\end{tabular}

Table 4 Elandt-Johnson's 'goodness of fit' to autosomal recessive inheritance, $\theta=0 \cdot 25$, including individuals with both complete and incomplete Behçet's syndrome as affected

\begin{tabular}{lrll}
\hline $\mathrm{Z}^{2}{ }_{\text {total }}$ & $11 \cdot 14$ & df 6 & Not significant \\
$\mathrm{Z}^{2}$ & 8.43 & df 1 & $* * *, \mathrm{p}<0 \cdot 01$ \\
$\mathrm{Z}^{2}{ }_{\text {diff }}$ & $2 \cdot 71$ & df 5 & Not significant \\
\hline
\end{tabular}

most frequent and problematical in individuals who had no other sign of Behçet's syndrome but were related to patients with Behçet's syndrome. When mouth and genital ulcers were included the number of families was reduced to 19 since five families had one parent with isolated mouth or genital ulcers. The model for an autosomal recessive being used will only fit families of marriages between unaffected, presumptive heterozygotes. Of these 19 families, 27 children had either Behçet's syndrome or mouth/genital ulcers. Table 5 shows the results of the analysis; surprisingly, $Z^{2}$ diff was not significant and showed no heterogeneity; these symptoms, at least, present in the same pattern as Behçet's syndrome itself in families. $Z^{2}$ comb was not now significant, suggesting a fit to the autosomal recessive model. This must be treated with caution, however, since $(a)$ the sample was biased by the exclusion of families where parents exhibited
Table 5 Elandt-Johnson's 'goodness of fit' to autosomal recessive inheritance, $\theta=0 \cdot 25$, including symptoms $M U$ and $G U$ as well as Behçet's syndrome as affected

\begin{tabular}{lccc}
\hline 19 families, 27 'affected' & & \\
$\mathrm{Z}^{2}$ total & $2 \cdot 89$ & df 6 & NS \\
$\mathrm{Z}^{2}$ comb & $0 \cdot 58$ & df 1 & NS \\
$\mathrm{Z}_{\text {diff }}^{2^{2}}$ & $2 \cdot 32$ & df 5 & NS
\end{tabular}

symptoms, and (b) the model did not, for instance, distinguish in this case between the conventional situation when both parents are heterozygous for the same allele and affected individuals are homozygous, and the situation where parents are heterozygous for different alleles at different loci and the affected individual is a double heterozygote. This latter model may be used to represent the interaction between predisposing HLA alleles and precipitating genetic factors in genetic explanations of HLA associated diseases.

Analysis of HLA antigen frequencies showed no significant differences between relatives and patients, or between symptomatic and non-symptomatic relatives. There was a suggestive absence of $\mathrm{A} 3$ in the UK patients $(0 / 7)$ compared with their relatives $(5 / 22)$, and this led to a re-examination of previously published data on HLA associations in the UK. ${ }^{12}$ 
Table 6 Frequencies of HLA antigens in patients with Behçet's syndrome and in controls, showing all significant associations in Turkey and the UK, and the B51 associations

\begin{tabular}{llll}
\hline & Patients & Controls & Fishers exact probability \\
\hline UK & $\mathrm{n}=14$ & $\mathrm{n}=34$ & \\
A3 & 0 & 9 & $0.0131^{*} \mathrm{p}<0.05$ \\
B27 & 4 & 1 & $0.021{ }^{*} \mathrm{p}<0.05$ \\
B51 & 3 & 4 & $0.327 \mathrm{NS}$ \\
Turkey & $\mathrm{n}=22$ & $\mathrm{n}=52$ & \\
B51 & 18 & 12 & $0 .(0)(0)(0) 344^{*} \mathrm{p}<0.01$ \\
\hline
\end{tabular}

Table 6 shows all the significant values of Fisher's exact probability of getting these ratios, relevant numbers of patients and controls, and an examination of the B51 frequencies in Turkey and the UK. It is interesting that in the $\mathrm{UK} \mathrm{A} 3$ is probably negatively associated with B5 (there is a precedent for 'protecting antigens' in diabetes mellitus). ${ }^{13} 14$ There may be an association with B27 in the UK, confirming earlier reports, ${ }^{815}$ and while B51 is certainly associated with Behçet's syndrome in Turkey, a probability of $32 \%$ suggests that there is no evidence for its being associated with Behçet's syndrome in the UK.

\section{Discussion}

The relatively small numbers of patients with Behçet's syndrome in the UK have made investigation of genetic hypotheses, particularly that of the rare autosomal recessive inheritance, difficult. The difficulty of diagnosis has further contributed to this problem. The technique applied here to $24 \mathrm{UK}$ and Turkish families, with 27 patients and 119 other relatives, has produced three major findings.

Firstly, the pattern of sporadic cases with unaffected parents recorded by Aoki, ${ }^{1}$ and noted here also, does not fit an autosomal recessive mode of inheritance.

Secondly, and perhaps more importantly, there is no apparent heterogeneity within the UK and Turkish populations, or between them. Whatever causes Behçet's syndrome affects individuals in families in the same way in both countries.

Thirdly, if the sample is biased by the inclusion of individuals with mouth and genital ulcers the heterogeneity does not increase significantly, suggesting that these symptoms in first degree relatives and patients with Behçet's syndrome may be related to the presence of the disease in some way.

These results led us to reconsider the relationship between histocompatibility antigens in patients, their relatives, and unrelated controls. We found no significant difference between patients and their relatives, but a suggestive absence of $\mathrm{A} 3$ in patients compared with their relatives. A reanalysis of previously published HLA frequencies in patients and controls by a more suitable statistical test leads to three conclusions. It is possible that A3 may be negatively associated with Behçet's syndrome in the UK. There is apparently no association of B51 with the syndrome in the UK. though the B51 association in Turkey is confirmed. There is a suggestion that B27 is associated with Behcet's syndrome in the UK. confirming earlier reports, ${ }^{8} 15$ though no association is shown between B27 and the syndrome in Turkey.

The exclusion of a simple genetic hypothesis for the aetiology of Behçet's syndrome does not imply that no genetic factors are involved. Nevertheless, at present it would seem to indicate that an examination of the mechanisms by which particular HLA antigens apparently predispose patients to illness is likely to be more productive in the search for an aetiology of Behçet's syndrome than further pedigree analysis.

I am very grateful to Dr M A Chamberlain. Professor H Yazici. and $\operatorname{Dr} M$ Denman for making available the data used in this analysis.

\section{References}

1 Aoki K. Ohno S, Ohguchi M. Sugiura S. Familial Behçet's disease. Jpn J Clin Opthalmol 1978; 22: 72-5.

2 Mason R M. Barnes C G. Behçet's syndrome with arthritis. Ann Rheum Dis 1969: 28: 95-103.

3 Goolamali S K. Comaish J S. Hassanyeh F. Stephens A. Familial Behçet's syndrome. Br J Dermatol 1976: 95: 637-42.

4 Berlin C. Behçet's disease as a multiple symptom complex. Arch Dermatol 1960): 82: 127-33.

5 Forbes I J. Robson H N. Familial recurrent orogenital ulceration. $\mathrm{Br}$ Med J 1960): i: 599.

6 Fowler T. Hamston D J. Nussey A M. Small M. Behçet's syndrome with neurological manifestations in two sisters. $\mathrm{Br}$ Med J 1968: ii: 473-4.

7 Fadli M E. Youssef M M. NeuroBehçet's syndrome in the United Arab Republic. Eur Neurol 1973; 9: 76.

8 Chamberlain M A. A family study of Behçet's syndrome. Ann Rheum Dis 1978: 37: 459-65.

9 O'Duffy J D. Lehner T. Barnes C G. Summary of the Third International Conference on Behçet's disease. Tokyo. Japan. October 23-24 1981. J Rheumatol 1983; 10: 1.

10 National Research Committee on Behçet's disease in Japan. Jpn J Opthalmol 1974: 18: 291-4.

11 Elandt-Johnson R. Probability models and statistical methods in genetics. New York: Wiley. 1971: 474-9.

12 Yazici H. Chamberlain M A. Schreuder I. D'Amaro J, Muftuoglu M. HLA antigens in Behçet's disease: a reappraisal by a comparative study of Turkish and British patients. Ann Rheum Dis 1980; 39: 344-8

13 Cudworth A G, Felsenstein H. HLA genetic heterogeneity in diabetes mellitus. Br Med Bull 1978; 34: 285-98.

14 Cudworth A G. Type 1 diabetes mellitus. Diabetologia 1978; 14: 281-91.

15 Takano M. Miyajima Y. Kiuchi M. Behçet's disease and the HLA system. Tissue Antigens 1976; 8: 95-9. 